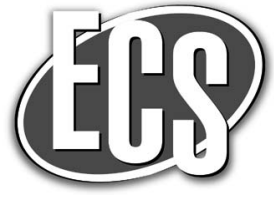

\title{
Kinetic Analysis of GaN-MOVPE via Thickness Profiles in the Gas Flow Direction with Systematically Varied Growth Conditions
}

\author{
Takeshi Momose, ${ }^{\mathrm{a}, \mathrm{z}}$ Tatsuya Kamiya, ${ }^{\mathrm{a}}$ Yudai Suzuki, ${ }^{\text {a }}$ Stefano Ravasio, ${ }^{\mathrm{b}}$ Carlo Cavallotti, \\ Masakazu Sugiyama, ${ }^{\mathrm{c}}$ and Yukihiro Shimogaki ${ }^{\mathrm{a}}$
}

\author{
${ }^{a}$ Department of Materials Engineering, The University of Tokyo, Bunkyo-ku, Tokyo 113-8656, Japan \\ ${ }^{b}$ Department of Chemistry, Materials and Chemical Engineering, Politecnico Di Milano, Bagnatica, \\ Bergamo 24060, Italy \\ ${ }^{c}$ Department of Electrical Engineering and Information Systems, The University of Tokyo, Tokyo 113-8656, Japan
}

\begin{abstract}
We carried out a kinetic analysis of metallorganic vapor phase epitaxy (MOVPE) of GaN to investigate the dependence of the growth rate on the process conditions as a function of residence time of the precursors in the reactor. The wafer was not rotated during growth, allowing us to analyze the thickness profile of the film in the direction of gas flow, and hence the dependence of the growth rate on the residence time. The growth rate is determined mainly by the concentration of the growth species and mass transfer of the growth species to the wafer surface. The growth rate peaked in the flow direction, and the position of this peak could, in most cases, be explained by considering a combination of the linear gas velocity and the time constant for vertical diffusion of trimethylgallium (TMGa) and/or growth species across the $\mathrm{NH}_{3}$ feed stream to the wafer surface. In some cases this was not possible, indicating that more complex effects were significant. This work is expected to contribute to understanding of the reaction pathways for GaN-MOVPE, and the growth rate data reported here are expected to provide useful benchmarks for growth simulations that combine computational fluid dynamics and reaction models.

(C) The Author(s) 2016. Published by ECS. This is an open access article distributed under the terms of the Creative Commons Attribution Non-Commercial No Derivatives 4.0 License (CC BY-NC-ND, http://creativecommons.org/licenses/by-nc-nd/4.0/), which permits non-commercial reuse, distribution, and reproduction in any medium, provided the original work is not changed in any way and is properly cited. For permission for commercial reuse, please email: oa@electrochem.org. [DOI: 10.1149/2.0071603jss] All rights reserved.
\end{abstract}

Manuscript submitted November 17, 2015; revised manuscript received December 16, 2015. Published January 2, 2016.

Gallium nitride $(\mathrm{GaN})$ is a III/V compound semiconductor material with considerable potential for optoelectronic and high-power electronic devices due to its wide bandgap and high breakdown voltage. ${ }^{1-5}$ For these reasons, $\mathrm{GaN}$ is currently used for mass-produced light-emitting diodes (LEDs), lasers, and high-frequency devices. ${ }^{6-10}$ Metalorganic vapor phase epitaxy (MOVPE) is commonly employed to manufacture $\mathrm{GaN}$ films using trimethylgallium (TMGa) and $\mathrm{NH}_{3}$ as group-III and group-V precursors, respectively. Research into the growth mechanisms involved in GaN-MOVPE in both academic and industrial institutions has found that the reaction chemistry is relatively complicated, consisting of gas-phase reactions followed by surface reactions. ${ }^{11-17}$ This intrinsic complexity of the reactions suggests that it is not straightforward to design optimal reactors for mass production as well as settling the optimal growth conditions via conventional empirical approaches. Therefore, a variety of studies have been carried out with the aim of understanding GaN-MOVPE. Initially, attention mainly focused on identification of intermediate species that are generated from the gas-phase precursors, which contribute to the layer growth. It then became apparent that parasitic reactions in the gas phase resulted in the formation of adducts, even in the non-heated area of the reactor, in addition to those formed in the hot zone in the vicinity of the heated substrate. ${ }^{18,19}$ Some of these reaction products led to particle formation without contributing to layer growth. ${ }^{20-22}$ Inlet configurations of reactors have been optimized to suppress these unwanted reactions before the precursors reach the growth surface. ${ }^{23-25}$ Developments in computer simulation technology have allowed us to analyze the elementary reactions numerically, and several reaction models have been reported, which were capable of reproducing experimental results with specific reactor configurations and growth conditions. ${ }^{26-33}$ Several plausible candidate growth species that contribute to layer growth have been reported, including TMGa: $\mathrm{NH}_{3}$ adducts, $\left[\left(\mathrm{CH}_{3}\right)_{2} \mathrm{GaNH}_{2}\right]_{3},{ }^{34-36}$ diatomic GaN, ${ }^{37,38}$ and $\mathrm{GaNH}_{2} \cdot{ }^{39}$ However, a model that can describe the growth behavior consistently in all reactor configurations and for all process conditions remains elusive. This is our concern, and necessitates comprehensive investigation on the behavior of $\mathrm{GaN}$ growth under a wide variety of conditions, from both experimental and theoretical standpoints.
With GaN-MOVPE, the reactor configuration and process conditions are usually designed to minimize the residence time of the precursors in the the reactor to suppress the above-mentioned parasitic reactions. Such a configuration makes the gas-phase reactions far from chemical equilibrium, so that the growth rate is largely determined by the reaction kinetics, not chemical equilibrium. ${ }^{39}$ Therefore, an analysis of the dependence of the growth behavior on the residence time is required. An analysis of the gas-phase species and the gasphase reactions is, however, extremely challenging due to spatial and temporal distributions of the gas-phase species within the reactor. However, it is possible to infer details of the growth kinetics from the profile of the growth rate in the gas flow direction. ${ }^{40}$ Analysis of film thickness profiles in the gas flow direction within longitudinal flow reactors has been used in a variety of chemical vapor deposition (CVD) systems. ${ }^{41-46}$ However, to date studies of GaN-MOVPE, especially the relation between the growth rate and the process conditions, have not considered this, and thus the growth rate has been investigated with respect to a fixed residence time; i.e., at a fixed position in the reactor. This means that there is a lack of systematic experimental evidence to benchmark GaN-MOVPE reaction models. Furthermore, with conventional GaN-MOVPE, the wafer is rotated to achieve an even film thickness and uniform crystallinity over the entire wafer; however, this masks details of the intrinsic reaction kinetics. For these reasons, we require experiments to obtain information relating to the details of the reaction kinetics.

Here, the wafer rotation was stopped following the growth of a template $\mathrm{GaN}$ layer, allowing us to obtain information on the film

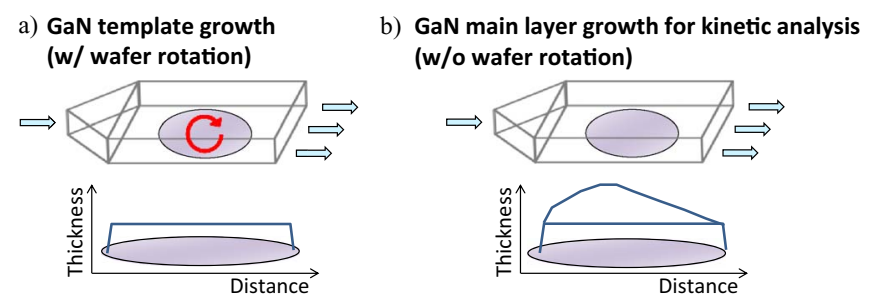

Figure 1. A schematic diagram of the gallium nitride $(\mathrm{GaN})$ growth process. (a) Template formation (with wafer rotation), and (b) target layer growth for kinetic analysis (without wafer rotation). 
a)

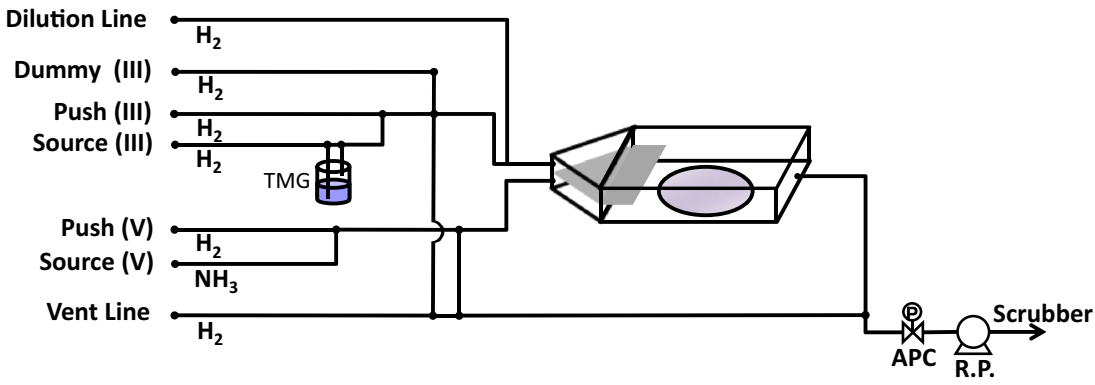

b)

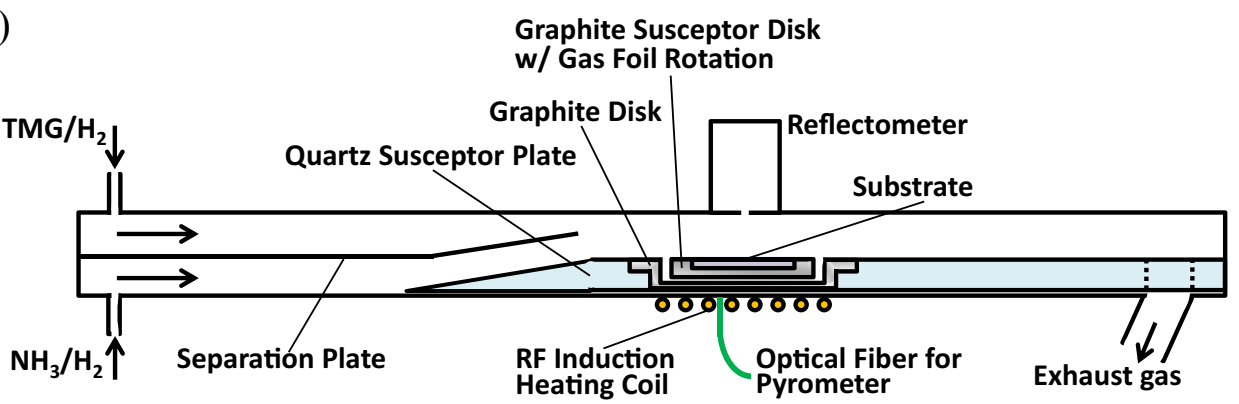

Figure 2. Schematic diagrams of the GaN MOVPE system. (a) The precursor supply system, and (b) a cross-section of the reactor.

thickness profiles and surface morphology in the flow direction, as shown in Fig. 1. Such an analysis is helpful for identifying the main reaction pathways and major species with the aid of computational fluid dynamics (CFD) simulations coupled with quantum chemistry calculations (which will be described in a future publication).

\section{Experimental}

GaN growth was carried out using a 2" sapphire wafer in a horizontal MOVPE reactor as shown in Fig. 2 (AIXTRON AIX 200RF-S). ${ }^{47-49}$ To determine the growth rate precisely, without being influenced by the initial nucleation behavior on the sapphire substrate, ${ }^{50-52} \mathrm{GaN}$ layers of a fixed thickness were grown using our standard recipe with a rotating wafer, which were then used as a template for the subsequent growth of a target $\mathrm{GaN}$ layer that was used for analysis of the growth kinetics. This target GaN layer was grown without rotating the wafer, which allowed us to investigate the growth rate profile in the direction of the flow of precursors, hence providing information on the dependence of the growth rate on the residence time of the precursors in the reactor. ${ }^{37,38,53}$ The growth conditions for the target $\mathrm{GaN}$ layer were systematically varied to analyze the dependence of the growth rate profile on the deposition conditions.

Trimethylgallium (TMGa) was used as the group-III precursor, with $\mathrm{NH}_{3}$ used as the group- $\mathrm{V}$ precursor, and $\mathrm{H}_{2}$ as the carrier gas. Two-inch single-side-polished sapphire wafers were used as a substrate. The growth conditions for the template layer were as follows: (i) a buffer layer with a V/III ratio of 2,548 was grown at $550^{\circ} \mathrm{C}$; (ii) a nucleation layer with a low V/III source ratio of 466 was grown at $1130^{\circ} \mathrm{C}$; and (iii) a template layer with a V/III ratio of 728 was grown at $1130^{\circ} \mathrm{C}$. Table I lists the conditions for growth of the $\mathrm{GaN}$ target layer. With the standard conditions, the time to reach the hot zone from the inlets was $0.23 \mathrm{~s}$, and the residence time within the hot zone was also $0.24 \mathrm{~s}$, which follows from considerations of the reactor configuration assuming cold walls. The growth conditions discussed in Substrate temperature section-H2 partial pressure with constant partial pressures of TMGa and $\mathrm{NH} 3$ section are also listed in Table I. The deposition period was $20 \mathrm{~min}$ in all cases. To inspect the growth progress in situ, the surface reflectivity at a wavelength of $951.3 \mathrm{~nm}$ was monitored throughout the growth period, including the growth of the buffer layer, using a reflectometer (AIXTRON

Table I. The conditions during growth of the target gallium nitride (GaN) layer.

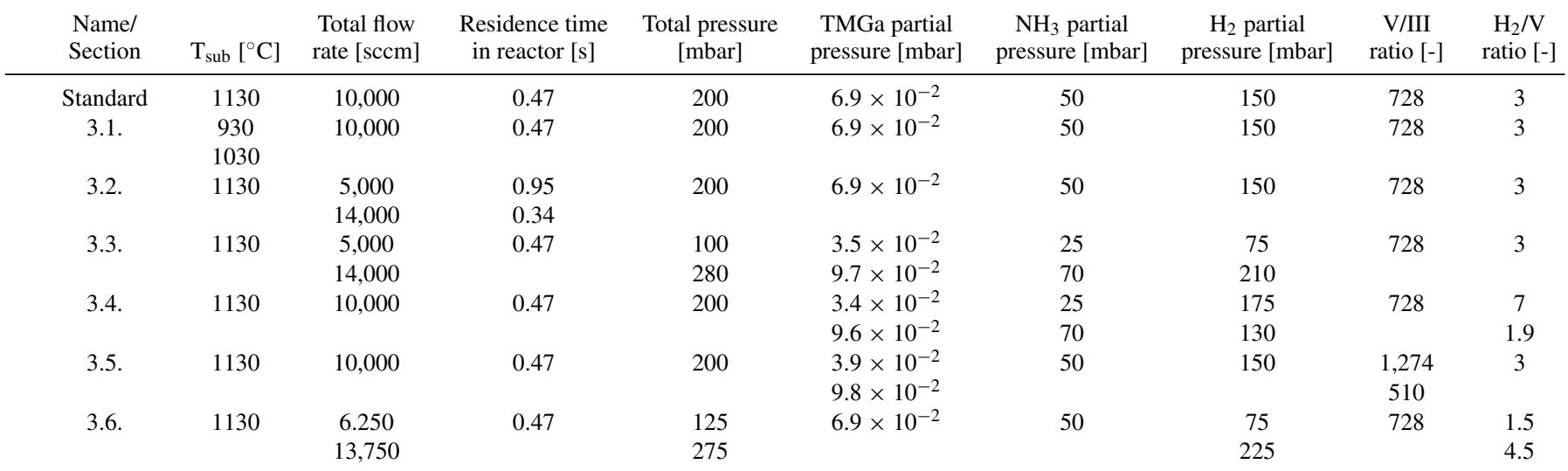


Epinet-Curve) installed above the center of the wafer. Because the initial nucleation and subsequent template growth is strongly dependent on the initial conditions of the wafer surface, and the template thickness after a fixed period was not necessarily constant for each run, reproducibility of the template thickness was enhanced using transient reflectivity curves. In other words, the template growth was terminated when the reflectivity reached the second peak, leading to template layers that were approximately $800-\mathrm{nm}$-thick. Reflectivity measurements were also employed to monitor the film thickness at the center of the wafer in situ during the growth of the target GaN layer. The film thickness as evaluated using in situ reflectivity measurements at the 25 -mm position (i.e., the wafer center), with a deviation of \pm 2.5 $\mathrm{mm}$ in the lateral direction considering misalignment of the laser irradiation spot is shown by the circular dots in Figs. 3-8. The film thickness profile in the gas-flow direction was measured ex situ at 5-mm intervals using an ultraviolet (UV) light source (Hamamatsu, L10290) and interferometry (EPSON, Filmetrics F20-UVX). The template thickness was then subtracted from overall film thickness, yielding the thickness of the target GaN layer. The surface morphology of the films was observed using Nomarski microscopy (Nikon, Eclipse ME600).

\section{Results and Discussion}

Substrate temperature.-Growth rate profiles were obtained as a function of the substrate temperature, which was varied in the range 930- $1130^{\circ} \mathrm{C}$ (see Fig. 3). All other conditions remained identical to the standard conditions (see Table I). Note that deposition at temperatures above $1180^{\circ} \mathrm{C}$ resulted in a hazy surface over the entire wafer, and therefore these wafers were not used in this study (data not shown). The growth rate at the upstream area increased with position in the flow direction in all cases, which is consistent with a discussion of the reaction mechanisms of GaN MOVPE, whereby GaN grows via gas-phase intermediate species generated from TMGa. However, such variation in the growth rate according to position in the flow direction may also result from vertical diffusion of both TMGa and of the gasphase species that is originated by TMGa across the feed stream of $\mathrm{NH}_{3}$, since the TMGa inlet was $18.6 \mathrm{~mm}$ above the surface of the wafer.

In the following, a rational explanation is given for the interpretation of profiles of the growth rate measured at the upstream area. The rate-limiting step in GaN-MOVPE is diffusion of the growth species. ${ }^{54}$ The growth rate depends on the product of the mass transfer coefficient $k_{d}$ and the concentration of growth species or gas-phase

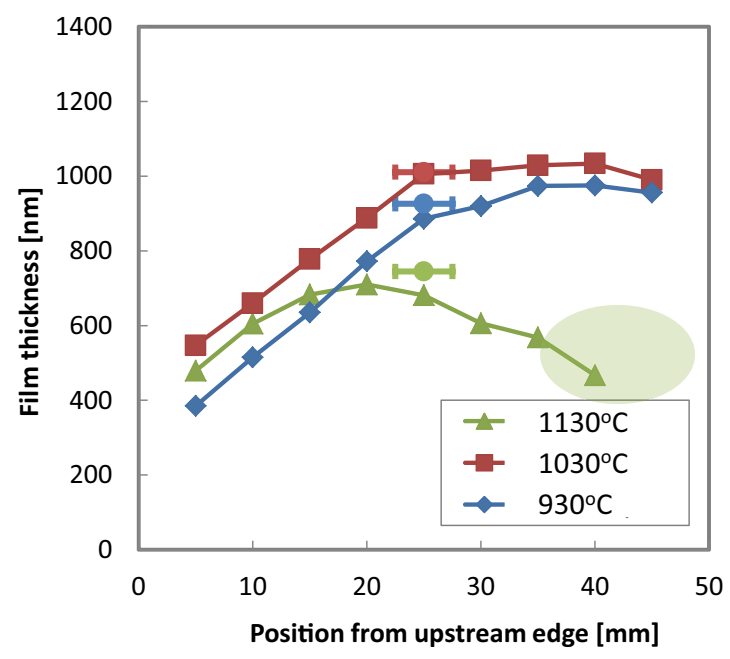

Figure 3. Film thickness as a function of position in the flow direction with various substrate temperatures. The shaded area corresponds to the regions with the hazy surface. Data were obtained from in situ reflectivity measurements (filled circles) and ex situ interferometry measurements (all other symbols). intermediates $C_{g s}$. The mass transfer coefficient can be expressed as $k_{d}=D / \delta$, where $D$ is the diffusion coefficient of the growth species, and $\delta$ is the thickness of the boundary layer. ${ }^{55} \mathrm{~A}$ hot zone that appears in the vicinity of the heated substrate ${ }^{32,39}$ may affect both $C_{g s}$ and $D$ for the growth species; however, the temperature dependence of $D$ is weak, ${ }^{56}$ and so the assumption that the composition profile in the upstream part is independent of temperature is reasonable if the growth is controlled by mass transport. The temperature of the hot zone will increase with increasing substrate temperature, increasing the rate of gas-phase reactions. This leads to more rapid generation of growth species in the gas phase, provided that the rate-limiting step is the gas-phase reaction to generate the growth species. A higher substrate temperature may therefore be expected to result in a faster growth rate; however this is not the case, and the independence of the growth rate from temperature indicates that mass transport of TMGa-related species from the inlet toward the growth surface is the rate-limiting step.

The decrease in growth rate in the downstream area at $1130^{\circ} \mathrm{C}$ may be attributable to nucleation of fine particles from the growth species in the gas phase, which is a parallel reaction pathway to layer growth. ${ }^{20-22}$ This was apparent at $1130^{\circ} \mathrm{C}$ due to the high temperature of the hot-zone caused by the hot substrate. This reasoning is consistent with hazy surface observed downstream (see the shaded area in Fig. 3). Here, the conversion efficiency of the precursor to the film was calculated from mass balance of the precursor within the reactor to evaluate the possibility that depletion of the precursor resulted in the decrease in the growth rate in the downstream direction. The TMGa molar flow rate was $1.5 \times 10^{-4} \mathrm{~mol} / \mathrm{min}$ and the growth period was $20 \mathrm{~min}$, which gives a total of $3.0 \times 10^{-3} \mathrm{~mol}$ of Ga atoms supplied into the reactor. The average film thickness with the highest growth temperature of $1130^{\circ} \mathrm{C}$ was $883 \mathrm{~nm}$, from which we may estimate that there were $1.2 \times 10^{-4} \mathrm{~mol}$ of Ga atoms on the $2^{\prime \prime}$ wafer, where a GaN density of $7.29 \times 10^{4} \mathrm{~mol} / \mathrm{m}^{3}$ was used in the calculations. The conversion efficiency of the precursor to the film was therefore $4 \%$, which means that an excess of precursor molecules would have remained in the downstream part of the reactor. Hence, it does not appear plausible that depletion of the precursors was the cause of the slower growth rate in the downstream part of the reactor.

Total flow rate.-The dependence of the growth rate on total flow rate was examined at $1130^{\circ} \mathrm{C}$. The total flow rate was in the range $5,000-14,000 \mathrm{sccm}$, and thereby the residence time of the precursors from the inlet to the end of the hot zone was in the range 0.95$0.34 \mathrm{~s}$. The other conditions were identical to the standard conditions described in the Experimental section (see Table I). As shown in Fig. 4, the peak in the film thickness shifted upstream with a smaller total flow rate; i.e., as the residence time increased. This can be explained by considering the progress of vertical diffusion of TMGarelated species and gas-phase reactions in the hot zone. With a smaller total flow rate, the residence time from the inlet to the wafer increased. This gave more time for both vertical diffusion and gas-phase reactions before the gases reached the upstream edge of the hot zone, leading to the peak growth rate occurring further upstream and the maximum growth rate increasing. To support this discussion, we plotted these data as a function of the residence time of the precursor from the inlet, as shown in Fig. 4b. The resulting location of the peak film thickness almost overlapped with flow rates between 10,000 and $14,000 \mathrm{sccm}$, and the maximum growth rate increased as the total flow rate increased, while those for 5,000 sccm could not be compared due to the peak missing. Minor difference of the peak position may be attributable to the temperature distribution in the vicinity of the heated substrate changing at different flow rates.

To enable a quantitative discussion, here we consider three values related to the mass transport phenomena of gas-phase species. The first is the diffusion coefficient of TMGa in a mixture of $\mathrm{NH}_{3}$ and $\mathrm{H}_{2}$. Here TMGa is considered to be representative of the chemical species that can be formed in the gas phase following TMGa decomposition, most notably $\mathrm{GaNH}_{2} \cdot{ }^{39}$ Because $\mathrm{GaNH}_{2}$ and $\mathrm{TMGa}$ have similar diffusion coefficients, we believe that this approximation is reasonable. We 

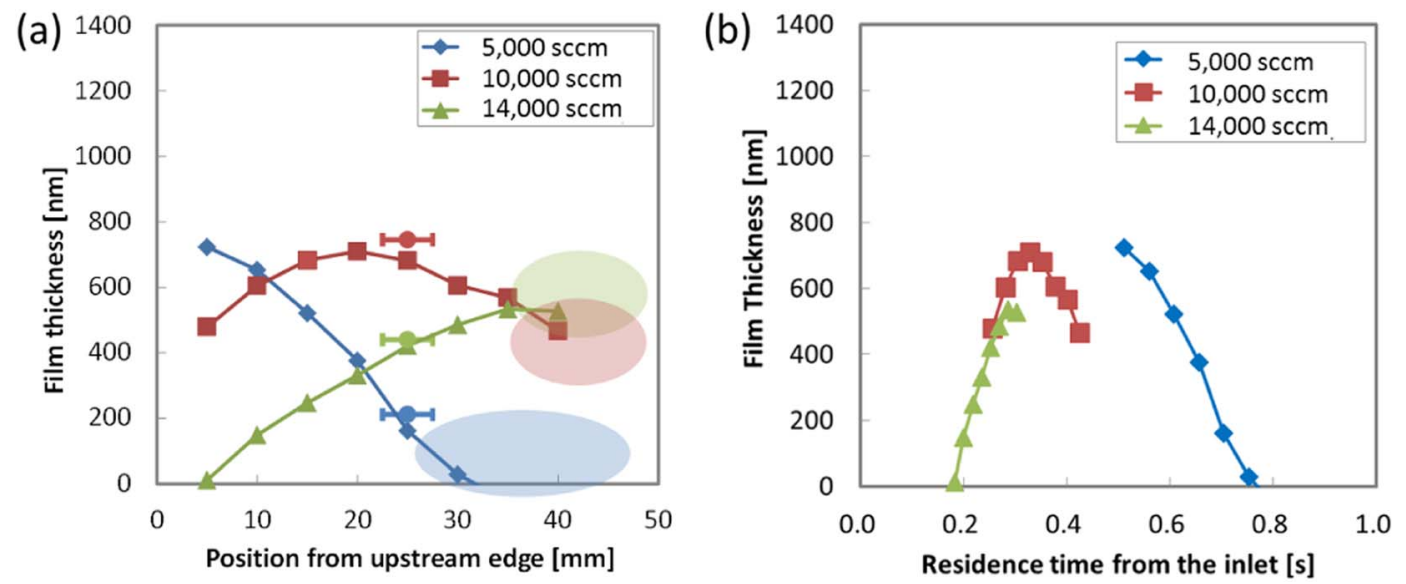

Figure 4. Film thickness profiles along the flow direction with different total flow rates. (a) As a function of the distance along the wafer from the upstream edge, and (b) as a function of the residence time of the inlet assuming the cold wall. The shaded areas correspond to positions with a hazy surface. Data were obtained using in situ reflectivity (filled circles) and ex situ interferometry (the other symbols).

calculated $D$ for TMGa in $\mathrm{NH}_{3}$ and in $\mathrm{H}_{2}$ individually using ChapmanEnskog theory, and then found $D$ in a $\mathrm{NH}_{3} / \mathrm{H}_{2}$ mixture as follows:

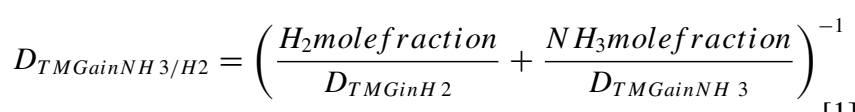

Table II lists the Lennard-Jones parameters of constituent species necessary for the Chapman-Enskog theory calculations.

The second parameter is the time constant $\tau$ for vertical diffusion of TMGa from the TMGa inlet to the growth surface. Since TMGa was supplied from the upper side of two stacked inlets, and $\mathrm{NH}_{3}$ was supplied from the lower side, TMGa must diffuse vertically in a mixture of $\mathrm{NH}_{3}$ and $\mathrm{H}_{2}$ to arrive at the growth surface. Assuming zero flow velocity in the vertical direction because of laminar flow, the concentration profile of TMGa along the vertical axis can be described using Fick's second law; i.e.,

$$
\frac{C(z, t)}{C_{0}}=1-\operatorname{erf}\left(\frac{z}{\sqrt{4 D t}}\right),
$$

\begin{tabular}{|c|c|c|}
\hline Species & $\sigma[\mathrm{A}]$ & $\varepsilon / \mathrm{k}_{\mathrm{B}}[\mathrm{K}]$ \\
\hline TMGa & 5.52 & 378.2 \\
\hline $\mathrm{H}_{2}$ & 2.92 & 38.0 \\
\hline $\mathrm{NH}_{3}$ & 2.92 & 481.0 \\
\hline
\end{tabular}

Abbreviation: TMGa, trimethylgallium

Table III. The mean position for $10 \%$ of the precursor to reach the growth surface with various total flow rates.

\begin{tabular}{ccccc}
$\mathrm{T}\left[{ }^{\circ} \mathrm{C}\right]$ & $\begin{array}{c}\text { Flow rate } \\
{[\mathrm{sccm}]}\end{array}$ & $\begin{array}{c}D_{\mathrm{TMGa}} \text { in } \mathrm{NH}_{3} / \mathrm{H}_{2} \\
\text { mixture }\left[\mathrm{m}^{2} / \mathrm{s}\right]\end{array}$ & $\tau[\mathrm{s}]$ & $\begin{array}{c}\text { Position on the } \\
\text { wafer }[\mathrm{mm}]\end{array}$ \\
\hline \multirow{2}{*}{200} & 5,000 & $2.41 \times 10^{-4}$ & 0.25 & 7 \\
& 10,000 & & & 33 \\
& 14,000 & & & -66 \\
300 & 5,000 & $3.44 \times 10^{-4}$ & 0.17 & -13 \\
& 10,000 & & & 21 \\
400 & 14,000 & & & 49 \\
& 5,000 & $4.61 \times 10^{-4}$ & 0.13 & -18 \\
& 10,000 & & & 6 \\
& 14,000 & & & 28
\end{tabular}

where $C_{0}$ is the TMGa concentration supplied to the reactor, $z$ is the vertical distance from the TMGa inlet to the surface, and $t$ is time. Here, we assumed no reactions of TMGa to estimate the dispersion of the total concentration of TMGa-related species in the vertical direction. In this manner, we calculated the nominal time for the TMGa-related species to reach the growth surface. Here, a diffusion length of $18.6 \mathrm{~mm}$ was used, which was the vertical separation between the TMGa inlet and the $\mathrm{NH}_{3}$ inlet in our set-up, as measured from the growth surface. Based on our finding that the film growth is controlled by diffusion, the nominal time $\tau$ was calculated using Eq. 2 to obtain $C$ (growth surface, $\tau$ ) / $C_{0}=0.1$, which is the time it takes a relevant amount of the precursor (10\%) to reach the growth surface.

The third parameter is the mean position on the wafer at which the precursor arrives. This was calculated from $\tau$ and the lateral component of the velocity of the feed gases within the reactor, considering that lateral distance between the end of the separation plate and the upstream edge of the wafer was $48 \mathrm{~mm}$. We assumed a uniform temperature of the feed gases, and three average temperatures used in the calculations; i.e., 200,300 , and $400^{\circ} \mathrm{C}$. In practice, the hot zone formed in the vicinity of the heated substrate will lead to a spatially varying temperature profile, and so the diffusivity will also vary, complicating an analytical approach. Table III lists these three transport properties for each set of conditions. All three of the experimentally measured peak positions could be described as using an average gas temperature of $300^{\circ} \mathrm{C}$, which is somewhat surprising as the temperature profile may be expected to influence many phenomena, including gas mixing and the linear velocity. Anyhow, our approach to use average and uniform temperature in place of actual temperature profile is an effective way to explain the results at a first level of approximation.

Total pressure with a constant composition of $\mathrm{TMGa}, \mathrm{NH}_{3}$ and $\boldsymbol{H}_{2}$. - The dependence of the growth rate on the total pressure was investigated for total pressures in the range 100-280 mbar, in which the ratio among partial pressures for $\mathrm{TMGa}, \mathrm{NH}_{3}$, and $\mathrm{H}_{2}$ was fixed and the concentration of these species increased in proportion to the total pressure. To provide a constant residence time, the total flow rate was adjusted in proportion to the total pressure. Hence, the difference in the growth rates results from the total pressure and the concentration of the reactants. The other conditions were the same as the standard conditions described in the experimental section (see Table I). As shown in Fig. 5, the resulting thicknesses were similar, regardless of the total pressure, but the peak shifted downstream as the total pressure increased. In the following, these two trends are explained considering global transport from the inlet to the wafer as discussed in the previous section, as well as local transport in the surface vicinity. 


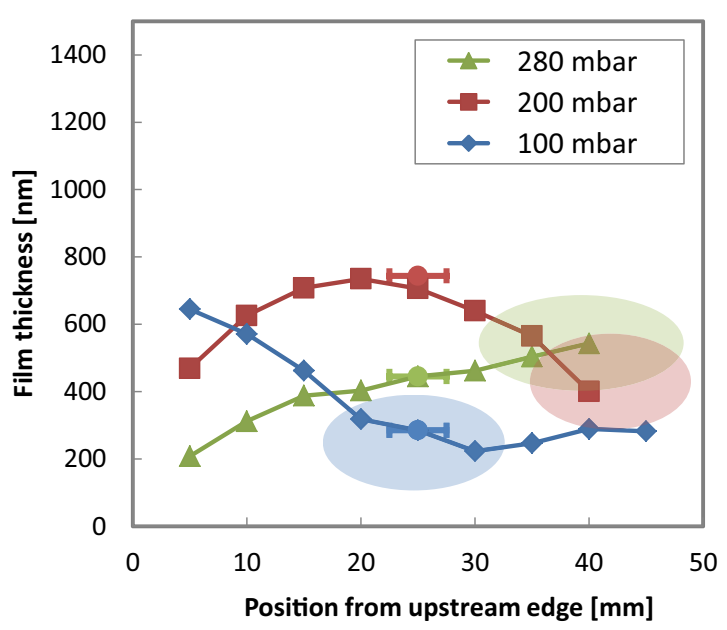

Figure 5. The film thickness profile in the flow direction for various total pressures. The precursor concentrations varied in proportion to the total pressure, and thus the V/III ratio was constant. Data were obtained via in situ reflectivity (filled circles) and ex situ interferometry (the other symbols).

\section{Table IV. The mean position for $10 \%$ of the precursor to reach the} growth surface with various total pressures.

\begin{tabular}{ccccc} 
T $\left[{ }^{\circ} \mathrm{C}\right]$ & $\begin{array}{c}D_{\mathrm{TMGa}} \text { in } \\
\mathrm{NH}_{3} / \mathrm{H}_{2} \\
{[\mathrm{mbar}]}\end{array}$ & $\begin{array}{c}\text { Predicted peak } \\
\text { mixture }\left[\mathrm{m}^{2} / \mathrm{s}\right]\end{array}$ & $\tau[\mathrm{s}]$ & $\begin{array}{c}\text { position on the } \\
\text { wafer }[\mathrm{mm}]\end{array}$ \\
\hline 300 & 100 & $6.88 \times 10^{-4}$ & 0.09 & -13 \\
& 200 & $3.44 \times 10^{-4}$ & 0.17 & 21 \\
& 280 & $2.46 \times 10^{-5}$ & 0.24 & 49
\end{tabular}

First, we describe the growth assuming that local transport phenomena in the vicinity of the wafer surface determine the growth rate. In the diffusion-limited regime, as discussed in Substrate temperature section, the growth rate can be expressed as the product of $k_{d}$ and $C_{g s}$. As $D$ is included in $k_{d}$ and is inversely proportional to the total pressure, and $\delta$ is independent of total pressure, $k_{d}$ is inversely proportional to the total pressure. On the other hand, $C_{g s}$ is proportional to the total pressure, as shown by the series of experiments described in this section (see Table I). Consequently, the overall pressure dependence cancels, and therefore we may expect the maximum growth rate to be independent of the total pressure. Second, we describe the growth assuming that global transport from the inlet to the wafer determined the growth position. The mean position at which $10 \%$ of the supplied precursor reaches the substrate was estimated (see Table IV). As $D$ is inversely proportional to the total pressure, $\tau$ increases as the total pressure increases, such that the peak may be expected to shift downstream as the total pressure increases. As with the previous section, a gas temperature of $300^{\circ} \mathrm{C}$ could be used to describe the observed peak positions.

TMG partial pressure with a constant V/III ratio.-The dependence of the growth rate on partial pressures of group-V and group-III sources was investigated with a constant V/III ratio. The partial pres-

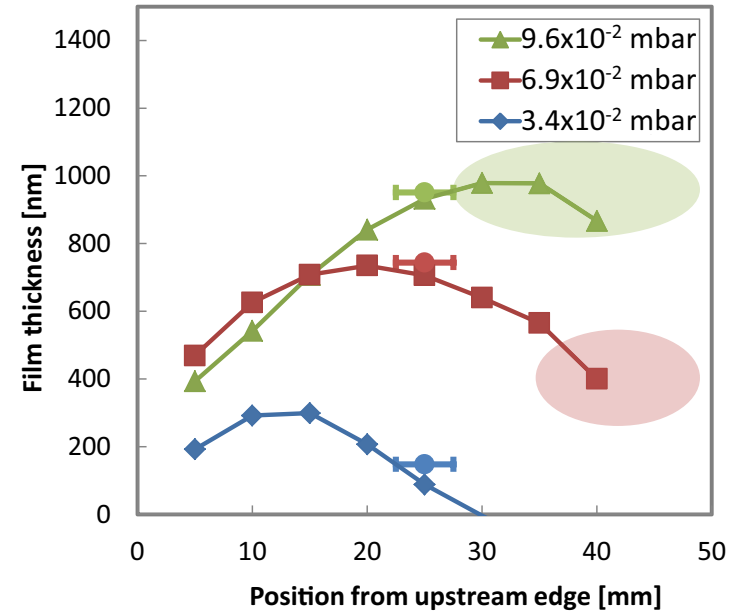

Figure 6. Film thickness profiles in the flow direction with various precursor concentrations with a constant V/III molar ratio. The legends in the graph denote the partial pressure of trimethylgallium (TMGa). Data were obtained using in situ reflectivity (filled circles) and ex situ interferometry (the other symbols).

sure of TMGa was varied from $3.4 \times 10^{-2}$ to $9.6 \times 10^{-2} \mathrm{mbar}$, and the partial pressure of $\mathrm{NH}_{3}$ was varied from 25 to 70 mbar. To maintain the residence time constant (and hence the total flow rate constant), the partial pressure of $\mathrm{H}_{2}$ was reduced as the partial pressure of TMGa increased. The other conditions were identical to the standard conditions described in the experimental section (see Table I). As shown in Fig. 6, as the TMGa concentration increased, the peak position shifted downstream and the maximum film thickness increased. These observations could be explained by considering the mechanisms discussed in Total flow rate section. As the partial pressure of TMGa increased, the growth rate increased. By contrast, an increase in the fraction of $\mathrm{NH}_{3}$ would lead to a decrease in $D$, and thereby a smaller $k_{d}$, and hence a decrease in the growth rate. The observation that the growth rate increased as the TMGa concentration increased suggests that the effect of the concentration of TMGa was stronger than the effects of $k_{d}$. The product of $D$ and the concentration of TMGa (see Table V) were positively correlated with the maximum growth rate, which supports the hypothesis that local transport phenomena in the vicinity of the surface determine the growth rate. However, there was some deviation of the peak growth rate from proportionality to the product of $D$ and the concentration of TMGa, indicating a more complex reaction mechanism.

The location of the peak growth rate was estimated from global transport from the inlet to the wafer by considering $D$ for the conditions listed in Table V. The calculated peak positions were in good agreement with the experiments. In the preceding sections, the molar ratio $\mathrm{NH}_{3} / \mathrm{H}_{2}$ was constant, and thus $D$ could be determined by the total pressure. However, here the molar ratio $\mathrm{NH}_{3} / \mathrm{H}_{2}$ affected $D$ even with a constant total pressure. Because of the larger $D$ for $\mathrm{H}_{2}$ than $\mathrm{NH}_{3}$, with a constant total pressure, at higher TMGa concentrations (i.e., lower $\mathrm{H}_{2}$ partial pressures) $D$ will be smaller for the mixed gases. As a result, as the concentration of precursor gases increased, the peak growth rate shifted downstream.

Table V. The mean position for $10 \%$ of the precursor to reach the surface with various precursor concentrations.

\begin{tabular}{|c|c|c|c|c|c|}
\hline $\mathrm{T}\left[{ }^{\circ} \mathrm{C}\right]$ & $\begin{array}{l}\text { TMGa partial } \\
\text { pressure [mbar] }\end{array}$ & $\begin{array}{l}D_{\mathrm{TMGa}} \text { in } \mathrm{NH}_{3} / \mathrm{H}_{2} \\
\text { mixture }\left[\mathrm{m}^{2} / \mathrm{s}\right]\end{array}$ & $\tau[\mathrm{s}]$ & $\begin{array}{l}\text { Predicted peak position } \\
\text { on the wafer [mm] }\end{array}$ & $\begin{array}{c}\text { Product of the TMGa partial } \\
\text { pressure and } D_{T M G a}\left[\mathrm{mbar} \cdot \mathrm{m}^{2} / \mathrm{s}\right]\end{array}$ \\
\hline \multirow[t]{3}{*}{300} & $9.6 \times 10^{-2}$ & $2.93 \times 10^{-4}$ & 0.20 & 33 & $2.8 \times 10^{-5}$ \\
\hline & $6.9 \times 10^{-2}$ & $3.44 \times 10^{-4}$ & 0.17 & 21 & $2.4 \times 10^{-5}$ \\
\hline & $3.4 \times 10^{-2}$ & $4.39 \times 10^{-4}$ & 0.14 & 6 & $1.5 \times 10^{-5}$ \\
\hline
\end{tabular}



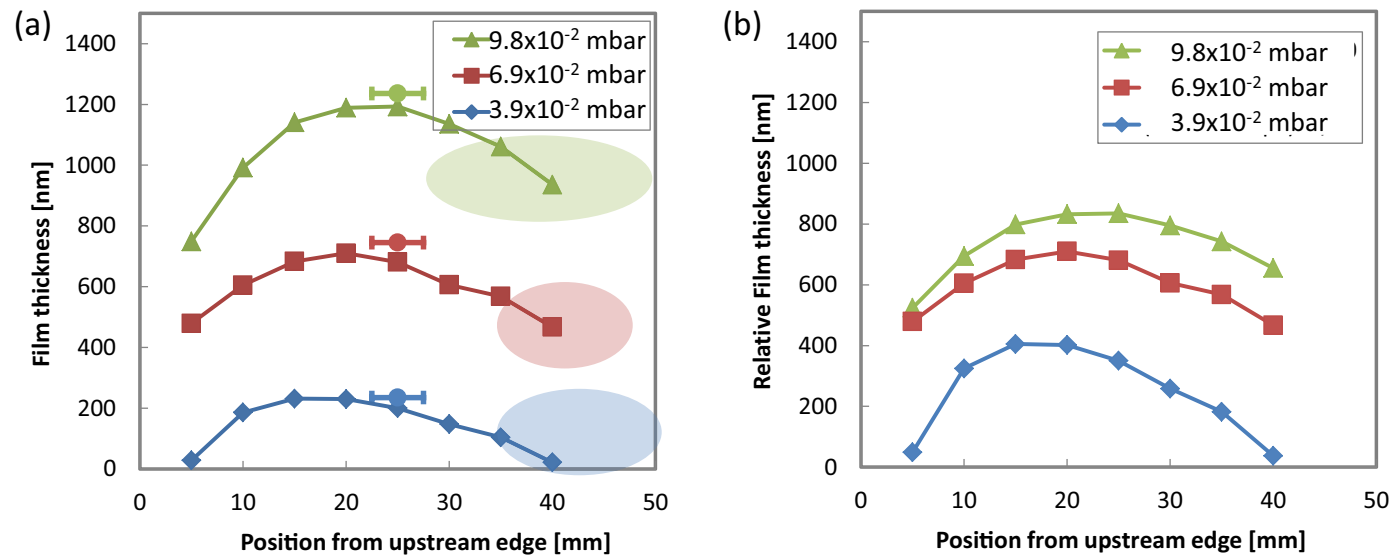

Figure 7. (a) Film thickness profiles in the flow direction with various TMGa partial pressures. (b) These data normalized to the TMGa partial pressure. The shaded areas correspond to a hazy surface of the wafer. Data were obtained using in situ reflectivity (filled circles) and ex situ interferometry (the other symbols).

TMGa partial pressure with constant $\mathrm{NH}_{3}$ partial pressure.The dependence of the growth rate on the partial pressure of TMGa was investigated with a constant partial pressure of $\mathrm{NH}_{3}$ to determine the dependence of the growth rate on the molar ratio of group- $\mathrm{V}$ to group-III precursors. The partial pressure of TMGa was varied from $3.9 \times 10^{-2}$ to $9.8 \times 10^{-2}$ mbar, and the partial pressure of $\mathrm{NH}_{3}$ was fixed, giving molar ratios V/III in the range 510-1,274. The other conditions were maintained as the standard conditions described in the experimental section (see Table I). As shown in Fig. 7, the results were in qualitative agreement with the mechanism discussed in section Total flow rate i.e., as the partial pressure of TMGa increased, so did the growth rate, whereas the location of the peak film thickness did not change significantly. Figure $7 \mathrm{~b}$ shows thickness profiles plotted so that the thickness was multiplied by the ratio of the partial pressures of TMGa; i.e., the film thicknesses with partial pressures of TMGa of $3.9 \times 10^{-2}$ and $9.8 \times 10^{-2}$ mbar were multiplied by $7 / 4$ and $7 / 10$, respectively. If all the reactions that occur during GaN-MOVPE obeyed linear reaction kinetics, these thickness profiles would overlap. However, the normalized thicknesses did not overlap, and furthermore the peak position shifted slightly downstream as the partial pressure of TMGa increased. It is evident that the variations in the peak positions and growth rates could not be explained using our model. On the other hand, our model could describe the data discussed in Total flow rate section-TMG partial pressure with a constant V/III ratio section. During those experiments, many experimental parameters were varied, including the total pressure and the partial pressures of TMGa, $\mathrm{NH}_{3}$ and $\mathrm{H}_{2}$ with a constant V/III ratio. Thus it can be inferred that some phenomena not considered in our model must depend on the V/III ratio.

$\mathrm{H}_{2}$ partial pressure with constant partial pressures of TMGa and $\mathrm{NH}_{3}$. - The dependence of the growth rate on the partial pressure of $\mathrm{H}_{2}$ was investigated for partial pressures of $\mathrm{H}_{2}$ in the range 75-225 mbar and constant partial pressures of TMGa and $\mathrm{NH}_{3}$, so that the total pressure varied from 125 to 275 mbar. To ensure that the residence time remained constant, the total flow rate was adjusted so that all gas flow rates were altered in proportion to the total pressure. The other conditions were identical to the standard conditions described in the experimental section (see Table I). As the partial pressure of $\mathrm{H}_{2}$ decreased, the peak growth rate shifted upstream, and the maximum film thickness increased. These observations could be explained in a similar manner as with the preceding sections.

Table VI lists calculated values of $D$ and the positions of the peak growth rate. First we discuss the behavior of the growth rate. The partial pressure of TMGa was constant, whereas $k_{d}$ decreased as the partial pressure of $\mathrm{H}_{2}$ increased because of the increase in the total pressure and decrease in $D$. As a result, the growth rate decreased as the partial pressure of $\mathrm{H}_{2}$ increased. This is reasonable be- cause the maximum growth rate was approximately proportional to $D$ (see Table VI). Calculations of the peak position were in good agreement with the experimental results, as with the preceding sections. A decrease in $D$ due to an increase in the partial pressure of $\mathrm{H}_{2}$ (as well as the total pressure) resulted in a downstream shift of the peak growth rate at higher total pressures.

On the basis of the data reported in Total flow rate section $-\mathrm{H}_{2}$ partial pressure with constant partial pressures of TMGa and $\mathrm{NH}_{3}$ section, here we discuss the contribution of the gas phase reactions to the film growth. In almost all cases, the growth rate could be described based on the product of $k_{d}$ and $C_{g s}$, so long as the molar ratio of group-V to group-III precursors remained constant (even though the partial pressures of TMG, $\mathrm{NH}_{3}$ and $\mathrm{H}_{2}$ were varied) (see Total flow rate section -TMG partial pressure with a constant V/III ratio section and $\mathrm{H}_{2}$ partial pressure with constant partial pressures of TMGa and $\mathrm{NH}_{3}$ section). However, when the molar ratio of group- $\mathrm{V}$ to group-III precursors changed (see TMGa partial pressure with constant $\mathrm{NH}_{3}$ partial pressure section), the growth rate could not be described based on the product of $k_{d}$ and $C_{g s}$, and the experimentally measured growth rate was smaller than that expected from the product of $k_{d}$ and $C_{g s}$ as the V/III ratio increased.

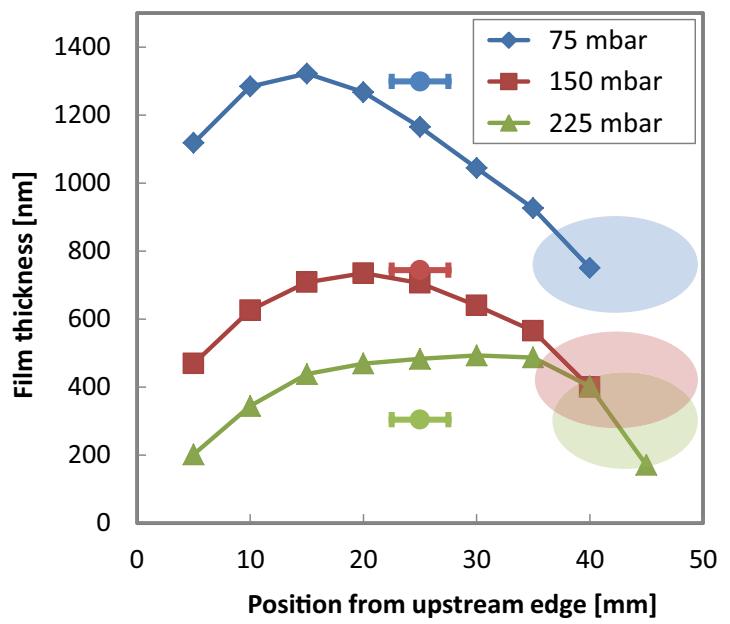

Figure 8. Film thickness profiles in the flow direction with various partial pressures of $\mathrm{H}_{2}$ (the carrier gas). The shaded areas correspond to positions on the wafer with a hazy surface. The precursor concentrations were constant, and the $\mathrm{H}_{2}$ partial pressure was varied to control the total pressure. Data were obtained using in situ reflectivity (filled circles) and ex situ interferometry (the other symbols). 
Table VI. The mean position for $10 \%$ of the precursor to reach the growth surface with various partial pressures of $\mathrm{H}_{2}$.

\begin{tabular}{|c|c|c|c|c|c|}
\hline $\mathrm{T}\left[{ }^{\circ} \mathrm{C}\right]$ & $\begin{array}{c}\mathrm{H}_{2} \text { partial } \\
\text { pressure [mbar] }\end{array}$ & $\begin{array}{c}D_{\mathrm{TMGa}} \text { in } \mathrm{NH}_{3} / \mathrm{H}_{2} \\
\text { mixture }\left[\mathrm{m}^{2} / \mathrm{s}\right]\end{array}$ & $\tau[\mathrm{s}]$ & $\begin{array}{l}\text { Predicted peak position } \\
\text { on the wafer [mm] }\end{array}$ & $\begin{array}{c}\text { Product of the TMGa partial } \\
\text { pressure and } D_{T M G a}\left[\mathrm{mbar} \cdot \mathrm{m}^{2} / \mathrm{s}\right]\end{array}$ \\
\hline \multirow[t]{3}{*}{300} & 75 & $4.37 \times 10^{-4}$ & 0.14 & 6 & $3.0 \times 10^{-5}$ \\
\hline & 150 & $3.44 \times 10^{-4}$ & 0.17 & 21 & $2.4 \times 10^{-5}$ \\
\hline & 225 & $2.84 \times 10^{-4}$ & 0.21 & 36 & $2.0 \times 10^{-5}$ \\
\hline
\end{tabular}

Abbreviation: TMGa, trimethylgallium

There are three phenomena that may explain this, which were not considered in our model. The first is that reverse reactions to generate the growth species from the precursor in the gas phase were promoted as the V/III ratio increased, leading to the occurrence of reactions with nonlinear kinetics, and resulting in the generation of growth species more slowly than expected. The second is that an excess of group- $\mathrm{V}$ sources $\left(\mathrm{NH}_{3}\right.$-related species) resulted in etching of the grown $\mathrm{GaN}$ film, which may be significant in certain operative conditions. ${ }^{57-60}$ The third is the formation of particles in the gas phase which, while not deposited on the substrate, may scavenge part of the supplied precursors before they reach the growth surface. The presence of such complicated phenomena during GaN-MOVPE cannot be described using a simple analytical approach. Instead, well-defined elementary reaction data can be used to explain these results; this is part of ongoing work, which will be described in a future publication.

\section{Semi-Quantitative Growth Model}

Figure 9 shows a schematic diagram of the growth mechanism. The position of the peak growth rate could be predicted by the global transport of the TMGa or its derivatives from the inlet to the wafer; i.e., by the product of $D$ and $\tau$ for each series of variations in the growth conditions, except for that described in TMGa partial pressure with constant NH3 partial pressure section, where the molar ratio of groupIII to group- $\mathrm{V}$ precursors was varied. $\mathrm{CFD}$ simulations are mandatory to investigate the effect of the V/III ratio on the transport phenomena. The dependence of the maximum growth rate on the experimental conditions could be explained within each series of experiments by the local transport in the boundary layer formed in the vicinity of the growth surface; i.e., using the product of $k_{d}$ and the partial pressure of TMGa in place of $C_{g s}$ (although the magnitude of growth rate could not be predicted), except for that described in TMGa partial pressure with constant $\mathrm{NH} 3$ partial pressure section, where the V/III ratio was varied. It suggests that, when the V/III ratio remains constant, $C_{g s}$ is proportional to the partial pressure of TMGa due to the linear reaction kinetics of the gas-phase reactions, while the variation in the V/III ratio with each set of conditions resulted in a variation in $C_{g s}$ in the gas phase. One suggestion here is that we should carefully consider the elementary reactions, especially the dependence on the V/III ratio. Furthermore, we focused on the position of the maximum growth rate, but the form of the profile was not discussed. The profile of the growth rate can be calculated using CFD simulations coupled with appropriate elementary reaction models.

\section{Conclusions}

Growth rate profiles of $\mathrm{GaN}$ in the gas flow direction were analyzed systematically to obtain insight into the reaction kinetics and transport phenomena in the MOVPE reactor. The reaction mechanism during GaN-MOVPE consists of consecutive processes starting from the gasphase reactions of precursors to form growth species, followed by the diffusion of these species and subsequent surface reactions, which is accompanied by a parallel process that results in the formation of particles. The location of the peak growth rate is determined largely by global transport phenomena in the reactor from the inlet to the wafer; i.e., by $D$ and $\tau$. The maximum growth rate was found to depend on the local transport in the surface vicinity; i.e., $k_{d}$ and $C_{g s}$, where the variation within each series of experiments depended on their product; accurate estimates of $C_{g s}$, which take into account the residence time, are mandatory to predict the growth rate.

The progress of gas-phase reactions is a key factor affecting $C_{g s}$ in the vicinity of the growth surface. Furthermore, when the ratio V/III increased, the growth rate was smaller than that expected from the product of $k_{d}$ and $C_{g s}$. Reproducing these details of the dependence of the growth rate on the process conditions requires CFD simulations based on an appropriate elementary reaction model; however, the systematic variation of the growth rate profiles obtained in this work is expected to contribute to the validation of such a reaction model. Intuitively, the semi-quantitative analysis of the growth rate profiles described here, in particular the location of the peak growth rate as well as the magnitude of the growth rate, provides important insight into the growth mechanisms during GaN-MOVPE.

\section{References}

1. Z. Z. Bandic, E. C. Piquette, P. M. Bridger, R. A. Beach, T. F. Kuech, and T. C. Mcgill, Solid State Electron., 42, 2289 (1998).

2. N. Q. Zhang, S. Keller, G. Parish, S. Heikman, S. P. Denbaars, and U. K. Mishra, IEEE Electron Device Lett., 21, 421 (2000).

3. S. Karmalkar and U. K. Mishra, IEEE Transactions on Electron Devices, 48, 1515 (2001).

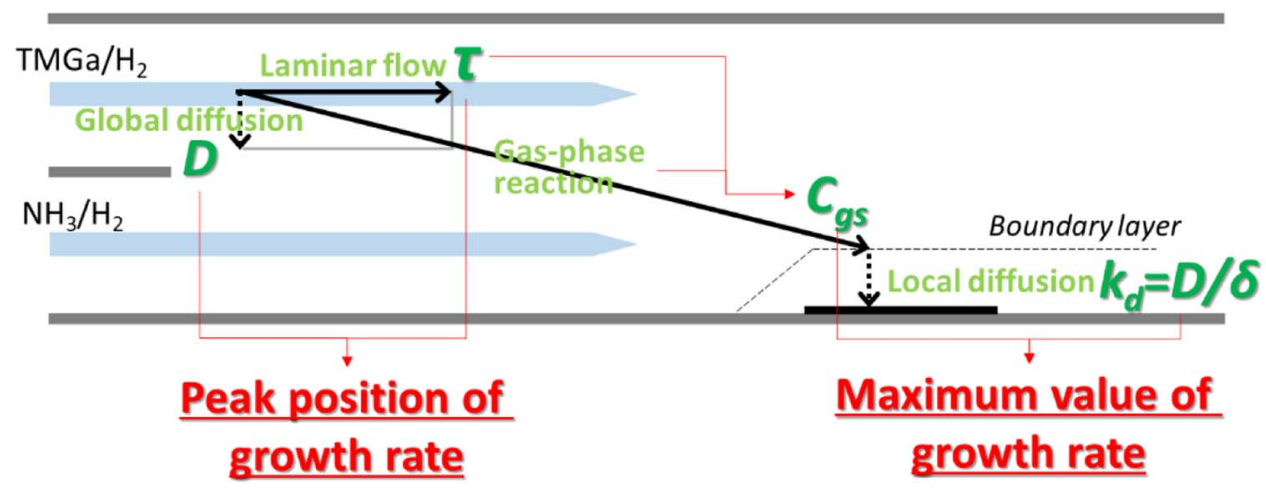

Figure 9. A schematic diagram showing the semi-quantitative reaction model used in this work. 
4. M. Higashiwaki, T. Mimura, and T. Matsui, Appl. Phys. Express, 1, 021103 (2008).

5. S. S. Lawrence, T. Suzue, and T. Egawa, IEEE Electron Device Lett., 30, 587 (2009).

6. M. A. Khan, A. Bhattarai, J. N. Kuznia, and D. T. Olson, Appl. Phys. Lett., 63, 1214 (1993).

7. Q. Chen, J. W. Yang, M. AsifKhan, A. T. Ping, and I. Aesida, Electron. Lett., 33, 1413 (1997).

8. Y. F. Wu, B. P. Keller, S. Keller, J. J. Xu, B. J. Thibeault, S. P. DenBaars, and U. K. Mishra, IEICE Trans. Electron., E82-C, 1895 (1999).

9. A. A. Burk, Jr.,, M. J. O'Loughlin, R. R. Siergiej, A. K. Agarwal, S. Sriram, R. C. Clarke, M. F. MacMillan, V. Balakrishna, and C. D. Brandt, Solid State Electron., 43, 1459 (1999).

10. Y. Ohno, IEEE Transactions on Electron Devices, 48,517 (2001).

11. D. Mazzarese, A. Tripathi, and W. C. Conner, J. Electro. Mater., 18, 3 (1989).

12. B. S. Sywe, J. R. Schlup, and J. H. Edgar, Chem. Mater, 3, 737 (1991).

13. S. H. Kim, H. S. Kim, J. S. Hwang, J. G. Choi, and P. J. Chong, Chem. Mater, 6, 278 (1994).

14. C. H. Chen, H. Liu, D. Steigerwald, W. Imler, C. P. Kuo, M. G. Craford, M. Ludowise, S. Lelster, and J. Amano, J. Electro. Mater, 25, 1004 (1996).

15. U. Bergmann, V. Reimer, and B. Atakan, Phys. Stat. Sol. A, 176, 719 (1999).

16. J. Schafer, A. Simons, J. Wolfrum, and R. A. Fischer, Chem. Phys. Lett., 319, 477 (2000)

17. J. Sun, J. M. Redwing, and T. F. Kuech, J. Electro. Mater, 29, 2 (2000).

18. S. A. Safvi, J. M. Redwing, M. A. Tischler, and T. F. Kuech, J. Electrochem. Soc., 144, 1789 (1997).

19. K. Matsumoto and A. Tachibana, J. Cryst. Growth, 272, 360 (2004).

20. J. R. Creighton, W. G. Breiland, and M. E. Coltrin, Electrochem. Soc. Proc., 2002-3, 28 (2002).

21. J. R. Creighton, G. T. Wang, W. G. Breiland, and M. E. Coltrin, J. Cryst. Growth, 261, 204 (2004).

22. A. Hirako and K. Ohkawa, J. Cryst. Growth, 289, 428 (2006).

23. C. Theodoropoulos, T. J. Mountziaris, H. K. Moffat, and J. Han, J. Cryst. Growth, 217, 65 (2000).

24. H. Hardtdegen, N. Kaluza, R. Schmidt, R. Steins, E. V. Yakovlev, R. A. Talalaev Y. N. Makarov, and J. T. Zettler, Phys. Stat. Sol. A, 201, 312 (2004).

25. H. Hardtdegen, N. Kaluza, R. Steins, R. Schmidt, K. Wirtz, E. V. Yakovlev, R. A. Talalaev, and Y. N. Makarov, J. Cryst. Growth, 272, 407 (2004).

26. K. Nakamura, O. Makino, A. Tachibana, and K. Matsumoto, J. Ogranomet. Chem., 611, 514 (2000).

27. K. Matsumoto and A. Tachibana, J. Cryst. Growth, 272, 360 (2004).

28. D. Sengupta, J. Phys. Chem. B, 107, 291 (2003).

29. D. Sengupta, S. Mazumder, W. Kuykendall, and S. A. Lowry, J. Cryst. Growth, 279, 369 (2005)

30. K. Kusakabe, A. Hirako, S. Tanaka, and K. Ohkawa, Phys. Stat. Sol. C, 10, 2569 (2004).

31. A. Hirako, K. Kuzakabe, and K. Ohkawa, Jpn. J. Appl. Phys., 44, 874 (2005).

32. D. Moscatelli, P. Caccioppoli, and C. Cavallotti, Appl. Phys. Lett., 86, 091106 (2005)

33. D. Moscatelli and C. Cavallotti, J. Phys. Chem. A, 111, 4620 (2007)
34. M. J. Almond, M. G. B. Drew, C. A. Jenkins, and D. A. Rice, J. Chem. Soc. Dalton Trans., 1, 5 (1992)

35. M. J. Almond, C. A. Jenkins, and D. A. Rice, J. Organomet. Chem., 439, 251 (1992).

36. A. Thon and T. F. Kuech, Appl. Phys. Lett., 69, 55 (1996)

37. M. Dauelsberg, C. Martin, H. Protzmann, A. R. Boyd, E. J. Thrush, J. Kappeler, M. Heuken, R. A. Talalaev, E. V. Yakovlev, and A. V. Kondratyev, J. Cryst. Growth, 298, 418 (2007)

38. M. Dauelsberg, D. Brien, R. Pusche, O. Schon, E. V. Yakovlev, A. S. Segal, and R. A. Talalaev, J. Cryst. Growth, 315, 224 (2011).

39. S. Ravasio, T. Momose, K. Fujii, Y. Shimogaki, M. Sugiyama, and C. Cavallotti, $J$ Phys. Chem. A, 119, 7858 (2015)

40. H. Komiyama, Y. Shimogaki, and Y. Egashira, Chem. Eng. Sci., 54, 1941 (1999).

41. Y. K. Chae, Y. Egashira, Y. Shimogaki, K. Sugawara, and H. Komiyama, Thin Solid Films, 320, 151 (1998)

42. Y. K. Chae, Y. Egashira, Y. Shimogaki, K. Sugawara, and H. Komiyama, J. Electroche. Soc., 146, 1780 (1999).

43. M. Sugiyama, H. Itoh, J. I. Aoyama, H. Komiyama, and Y. Shimogaki, Jpn. J. Appl. Phys., 39, 1074 (2000).

44. T. Saito, K. Oshima, Y. Shimogaki, Y. Egashira, K. Sugawara, K. Takahiro, S. Nagata, S. Yamaguchi, and H. Komiyama, Chem. Eng. Sci., 66, 6403 (2007).

45. T. Saito, K. Oshima, Y. Shimogaki, Y. Egashira, K. Sugawara, K. Takahiro, S. Nagata S. Yamaguchi, and H. Komiyama, Int. J. Chem. Reactor Eng., 10, A45 (2012).

46. Y. Fukushima, N. Sato, Y. Funato, H. Sugiura, K. Hotozuka, T. Momose, and Y. Shimogaki, ECS J. Solid State Science and Technol., 2, P492 (2013).

47. M. Sugiyama, S. Yasukochi, T. Shioda, Y. Shimogaki, and Y. Nakano, Phys. Status Solidi C, 7, 2085 (2010)

48. Y. Tomita, T. Shioda, M. Sugiyama, Y. Shimogaki, and Y. Nakano, J. Cryst. Growth, 311, 2813 (2009).

49. T. Shioda, M. Sugiyama, Y. Shimogaki, and Y. Nakano, Phys. Stat. Sol. A, 207, 1375 (2010).

50. I. Akasaki and H. Amano, J. Cryst. Growth 146, 455 (1995)

51. S. N. Mohammad and H. MorkocE, Progr. Quantum Electronics, 20, 361 (1996).

52. R. P. Parikh and R. A. Adomaitis, J. Cryst. Growth, 286, 259 (2006).

53. S. Y. Karpov, J. Cryst. Growth, 248, 1 (2003).

54. C. H. Chen, H. Liu, D. Steigerwald, W. Imler, C. P. Kuo, M. G. Craford, M. Ludowise, S. Lester, and J. Amano, J. Electro. Mater, 25, 1004 (1996).

55. R. B. Bird, W. E. Stewart, and E. N. Lightfoot, Transport Phenomena Revised Second Edition, John Wiley \& Sons, Inc., New York, USA 2007.

56. S. Sivaram, Chemical Vapor Deposition, Van Nostrand Reinhold, New York, USA 1995.

57. D. D. Koleske, A. E. Wickenden, R. L. Henry, J. C. Culbertson, and M. E. Twigg, J Cryst. Growth, 223, 466 (2001).

58. M. Mayumi, F. Satoh, Y. Kumagai, K. Takemoto, and A. Koukitu, J. Cryst. Growth, 237-239, 1143 (2002).

59. D. D. Koleske, M. E. Coltrin, A. A. Allerman, K. C. Cross, C. C. Mitchell, and J. J. Figiel, Appl. Phys. Lett., 82, 1170 (2003).

60. Y. H. Yeh, K. M. Chen, Y. H. Wu, Y. C. Hsu, T. Y. Yu, and W. I Lee, J. Cryst. Growth, 333, 16 (2011). 